\title{
iCoseg: Interactive Co-segmentation with Intelligent Scribble Guidance
}

\author{
Dhruv Batra \\ Carnegie Mellon Univerity \\ www. ece.cmu.edu/ dbatra
}

\author{
Adarsh Kowdle \\ Cornell University \\ apk64@cornell.edu
}

\author{
Devi Parikh \\ Toyota Technological Institute at Chicago (TTIC) \\ dparikhettic.edu
}

\author{
Jiebo Luo \\ Tsuhan Chen \\ Eastman Kodak Company \\ jiebo.luo@kodak.com \\ Cornell University \\ tsuhandece.cornell.edu
}

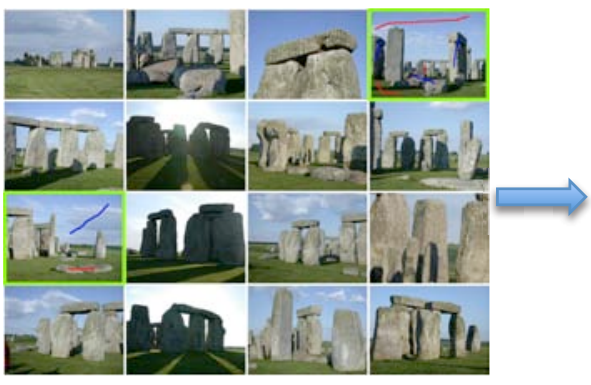

(a) Group of related images + multiple scribbles

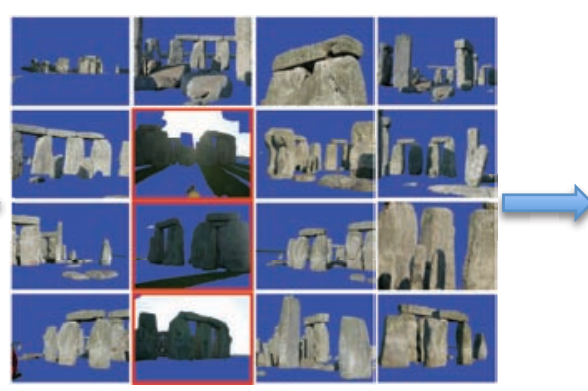

(b) Current segmentations

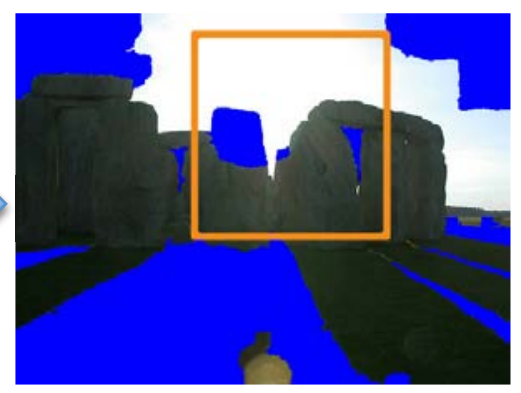

(c) Next scribble region recommendation

Figure 1. Overview of iCoseg: (a) shows a group of Stonehenge images, and foreground/background scribbles on two images (with green borders); (b) shows cutouts using these scribbles. A naïve interactive co-segmentation setup would force a user to examine all cutouts for mistakes, and then iteratively scribble on the worst segmentation to obtain better results. Cutouts needing correction are shown with red borders. (c) shows the region prompted for more scribbles by iCoseg, thus avoiding exhaustive examination of all cutouts by users.

\begin{abstract}
This paper presents an algorithm for Interactive Cosegmentation of a foreground object from a group of related images. While previous approaches focus on unsupervised co-segmentation, we use successful ideas from the interactive object-cutout literature. We develop an algorithm that allows users to decide what foreground is, and then guide the output of the co-segmentation algorithm towards it via scribbles. Interestingly, keeping a user in the loop leads to simpler and highly parallelizable energy functions, allowing us to work with significantly more images per group. However, unlike the interactive single image counterpart, a user cannot be expected to exhaustively examine all cutouts (from tens of images) returned by the system to make corrections. Hence, we propose iCoseg, an automatic recommendation system that intelligently recommends where the user should scribble next. We introduce and make publicly available the largest co-segmentation dataset yet, the CMUCornell iCoseg Dataset, with 38 groups, 643 images, and pixelwise hand-annotated groundtruth. Through machine experiments and real user studies with our developed in-
\end{abstract}

terface, we show that iCoseg can intelligently recommend regions to scribble on, and users following these recommendations can achieve good quality cutouts with significantly lower time and effort than exhaustively examining all cutouts.

\section{Introduction}

If there is one thing that the growing popularity of photosharing website like Flickr and Facebook (4 and 10 Billion photos respectively, as of Oct. 2009) has taught us - it is that people love taking photographs. Consumers typically have several related pictures of the same object, event or destination, and this rich collection is just waiting to be exploited by vision researchers - for something as simple as building a collage of all the foregrounds to something more sophisticated like a complete 3D model of a particular object. In many such tasks, it would be useful to extract a foreground object from all images in a group of related images. This co-segmentation of foreground objects from multiple related images is the goal of this paper. 

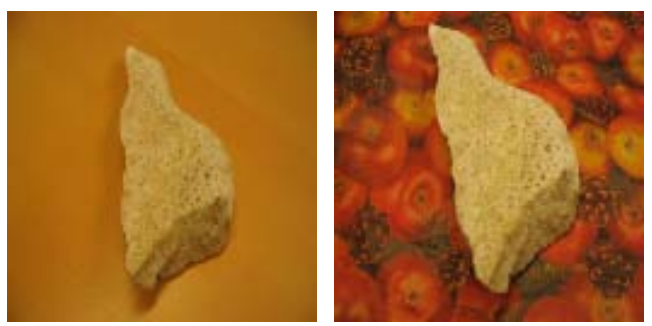

(a) Stone-pair [23].
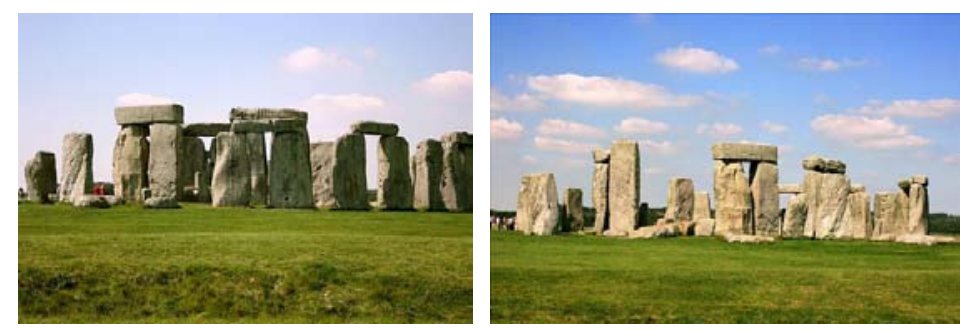

(b) Stonehenge-pair from CMU-Cornell iCoseg Dataset.

Figure 2: What is foreground? The stone-pair (a) has significant variation in background with nearly identical foreground and thus unsupervised co-segmentation can easily extract the stone as foreground. The Stonehenge-pair is fairly consistent as a whole and thus the stones cannot be cut out via unsupervised co-segmentation. Bringing a user in the loop is necessary for the problem of foreground extraction to be well defined.

Most existing works on co-segmentation [13, 21, 23] work with a pair of images with similar (sometimes nearly identical) foreground, and unrelated backgrounds (e.g. the "Stone-pair" in Figure 2). This property is necessary because the goal of these works is to extract the common foreground object automatically, without any user-input. Due to the nature of our application (i.e. multiple images of the same event or subject), our images typically do not follow this property (see Figure 2). Hence, without user-input, the task of extracting the foreground object "of interest" is illdefined.

This paper deals with Interactive Co-segmentation of a group (typically $\gg 2$ ) of related images, and presents an algorithm that enables users to quickly guide the output of the co-segmentation algorithm towards the desired output via scribbles. Our approach uses successful ideas from the single-image interactive segmentation $[6,19,22]$ literature. A user provides foreground/background scribbles on one (or more) images from a group and our algorithm uses these scribbles to produce cutouts from all images this group.

In a single-image setup, a user visually inspects the produced cutout and gives more scribbles to correct mistakes made by the algorithm. However, this approach would not work for interactive co-segmentation because 1) as the number of images in the group increases, it becomes increasingly cumbersome for a user to iterate through all the images in the group to find the worst segmentation; and 2) even if the user were willing to identify an incorrect cutout, there might be multiple incorrect cutouts in the group, some more confusing to the segmentation algorithm than others. Observing labels on the most confusing ones first would help reduce the number of user annotations required. It is thus necessary for the algorithm to be able to suggest regions in images where scribbles would be the most informative.

Contributions. The main contributions of this paper are:

- We present the first algorithm for intelligent Interactive
Co-segmentation (iCoseg), that automatically suggests regions where the user should scribble next.

- We introduce (and show results on) the largest cosegmentation dataset yet, the CMU-Cornell iCoseg dataset, containing 38 groups with 17 images/group on average (total 643 images) and pixelwise handannotated groundtruth. We make this dataset (and annotations) publicly available [3] to facilitate further work, and allow for easy comparisons.

- We develop a publicly available interface [3] for interactive co-sgementation. We present results of simulated machine experiments as well as real user studies on our interface. We find that iCoseg can intelligently recommend regions to scribble on, and help users achieve good quality cutouts with significantly lower time and effort than having to examine all cutouts exhaustively.

Technique. Our approach is composed of two main parts: 1) an energy minimization framework for interactive cosegmentation; and 2) a scribble guidance system that uses active learning and some intuitive cues to form a recommendation map for each image in the group. The system recommends a region with the highest recommendation score. See Figure 1 for an overview.

Organization. The rest of this paper is organized as follows: Section 2 discusses related work; Section 3 presents our energy minimization approach to interactive co-segmentation of a group of related images; Section 4 presents our recommendation scheme for guiding user scribbles; Section 5 introduces our benchmark dataset; Section 6 discusses the results of simulated machine experiments and a real user-study; Finally, Section 7 concludes the paper.

\section{Related Work}

Unsupervised Co-segmentation. Rother et al. [23] introduced the problem of (unsupervised) co-segmentation 
of image pairs. Their approach is to minimize an energy function that is a combination of the usual MRF smoothness prior and a histogram matching term that forces foreground histograms of images to be similar. $\mathrm{Mu}$ et al. [20] extend this framework with quadratic global constraints. More recently, Mukherjee et al. [21] proposed half-integrality algorithms, and Hochbaum et al. [13] modified the histogram matching term to propose max-flow based algorithms. The common theme here is unsupervised co-segmentation, which is achieved by forcing histogram consistency between foregrounds. As noted earlier, this would fail for pairs with related backgrounds (see Figure 2), where the problem of identifying the foreground objects is ill-posed. This is where our work of interactive co-segmentation fits in, which allows a user to indicate the foreground objects through simple scribbles. In addition, these works involve specific constructions and solutions for image pairs, while our technique naturally generalizes to multiple images (Section 3).

Supervised Co-segmentation. Schnitman et al. [24] and Cui et al. [12] learn to segment from a single fully segmented image, and then "induce" [24] or "transduce" [12] segmentations on a group of related images. We, on the other hand, utilize very sparse user interaction (in the form of scribles), which are not restricted to a single image and can be provided on multiple images in a group if desired.

Interactive Image Segmentation. Boykov and Jolly [6] posed interactive single-image segmentation given user scribbles as a discrete optimization problem. Li et al. [19] and Rother et al. [22] presented simplified user interactions. Bai et al. [2] and Criminisi et al. [11] proposed techniques built on efficient geodesic distance computations. Our approach to multiple-image interactive co-segmentation, as described in the next section, is a natural extension of Boykov and Jolly [6].

Active Learning. Related to our paper are works on active learning where algorithms are able to choose the data they learn from by querying the labelling oracle. This is a vast sub-field of machine learning and we refer the reader to Settles [25] for a detailed survey. In computer vision, active learning has been used for object categorization [15], classifying videos [28], ranking images by informativeness [27] and creating large datasets [9]. More recently, Kolhi et al. [16] showed how to measure uncertainties from graphcut solutions and suggested that these may be helpful in interactive image segmentation applications. To the best of our knowledge, this is the first paper to use uncertainties to guide user scribbles.

\section{3. iCoseg: Energy Minimization}

Energy Minimization. Given user scribbles indicating foreground / background, we cast our labelling problem as minimization of Gibbs energies defined over graphs constructed over each image in a group. Specifically, consider a group of $m$ image-scribble pairs $D=$ $\left\{\left(\mathcal{X}^{(1)}, \mathcal{S}^{(1)}\right),\left(\mathcal{X}^{(2)}, \mathcal{S}^{(2)}\right), \ldots,\left(\mathcal{X}^{(m)}, \mathcal{S}^{(m)}\right)\right\}$, where the $k^{\text {th }}$ image is represented as a collection of $n_{k}$ sites to be labelled, i.e. $\mathcal{X}^{(k)}=\left\{X_{1}^{(k)}, X_{2}^{(k)}, \ldots, X_{n_{k}}^{(k)}\right\}$, and scribbles for an image $\mathcal{S}^{(k)}$ are represented as the partial (potentially empty) ${ }^{1}$ set of labels for these sites. For computational efficiency, we use superpixels as these labelling sites (instead of pixels). ${ }^{2}$ For each image $(k)$, we build a graph, $\mathcal{G}^{(k)}=\left(\mathcal{V}^{(k)}, \mathcal{E}^{(k)}\right)$, over superpixels, with edges connecting adjacent superpixels.

Using these labelled sites, we learn a group appearance model $\mathcal{A}=\left\{A_{1}, A_{2}\right\}$, where $A_{1}$ is the first-order (unary) appearance model, and $A_{2}$ the second-order (pairwise) appearance model. This appearance model $(\mathcal{A})$ is described in detail in the following sections. We note that all images in the group share a common model, i.e. only one model is learnt. Using this appearance model, we define a collection of energies over each of the $m$ images as follows:

$$
\begin{aligned}
E^{(k)}\left(\mathcal{X}^{(k)}\right. & : \mathcal{A})=\sum_{i \in \mathcal{V}^{(k)}} E_{i}\left(X_{i}^{(k)}: A_{1}\right) \\
& +\lambda \sum_{(i, j) \in \mathcal{E}^{(k)}} E_{i j}\left(X_{i}^{(k)}, X_{j}^{(k)}: A_{2}\right),
\end{aligned}
$$

where the first term is the data term indicating the cost of assigning a superpixel to foreground and background classes, while the second term is the smoothness term used for penalizing label disagreement between neighbours. Note that the $(:)$ part in these terms indicates that both these terms are functions of the learnt appearance model. From now on, to simplify notation, we write these terms as $E_{i}\left(X_{i}\right)$ and $E_{i j}\left(X_{i}, X_{j}\right)$, and the dependence on the appearance model $\mathcal{A}$ and image $(k)$ is implicit.

Data (Unary) Term. Our unary appearance model consists of a foreground and background Gaussian Mixture Model, i.e., $A_{1}=\left\{\mathrm{GMM}_{f}, \mathrm{GMM}_{b}\right\}$. Specifically, we extract colour features extracted from superpixels (as proposed by Hoiem et al. [14]). We use features from labelled sites in all images to fit foreground and background GMMs (where number of gaussians was automatically learnt by minimizing an MDL criteria [5]). We then use these learnt GMMs to compute the data terms for all sites, which is the negative log-likelihood of the features given the class model.

Smoothness (Pairwise) Term. The most commonly used smoothness term in energy minimization based segmentation methods $[11,12,22]$ is the contrast sensitive Potts model:

$$
E\left(X_{i}, X_{j}\right)=\mathrm{I}\left(X_{i} \neq X_{j}\right) \exp \left(-\beta d_{i j}\right)
$$

\footnotetext{
${ }^{1}$ Specifically, we require at least one labelled foreground and background site to train our models, but only one per group, not per image.

${ }^{2}$ We use mean-shift [10] to extract these superpixels, and typically break down $350 \times 500$ images into 400 superpixels per image.
} 
where $\mathrm{I}(\cdot)$ is an indicator function that is $1(0)$ if the input argument is true(false), $d_{i j}$ is the distance between features at superpixels $i$ and $j$ and $\beta$ is a scale parameter. Intuitively, this smoothness term tries to penalize label discontinuities among neighbouring sites but modulates the penalty via a contrast-sensitive term. Thus, if two adjacent superpixels are far apart in the feature space, there would be a smaller cost for assigning them different labels than if they were close. However, as various authors have noted, this contrast sensitive modulation forces the segmentation to follow strong edges in the image, which might not necessarily correspond to object boundaries. For example, Cui et al. [12] modulate the distance $d_{i j}$ based on statistics of edge profile features learnt from a fully segmented training image.

In this work, we use a distance metric learning algorithm to learn these $d_{i j}$ from user scribbles. The basic intuition is that when two features (which might be far apart in Euclidean distance) are both labelled as the same class by the user scribbles, we want the distance between them to be low. Similarly, when two features are labelled as different classes, we want the distance between them to be large, even if they happen to be close by in Euclidean space. Thus, this new distance metric captures the pairwise statistics of the data better than Euclidean distance. For example, if colours blue and white were both scribbled as foreground, then the new distance metric would learn a small distance between them, and thus, a blue-white edge in the image would be heavily penalized for label discontinuity, while the standard contrast sensitive model would not penalize this edge as much. The specific choice of this algorithms is not important, and any state-of-art technique may be used. We use the implementation of Batra et al. [4].

We update both $A_{1}=\left\{\mathrm{GMM}_{f}, \mathrm{GMM}_{b}\right\}$ and $A_{2}=$ $\left\{d_{i j}\right\}$ every time the user provides a new scribble. Finally, we note that contrast-sensitive potts model leads to a submodular energy function. We use graph-cuts to efficiently compute the MAP labels for all images, using the implementation of Bagon [1] and Boykov et al. [7, 8, 17].

Comparing Energy Functions. Our introduced energy functions (1) are different from those typically found in cosegmentation literature and we make the following observations. While previous works [13, 20, 21, 23] have formulated co-segmentation of image pairs with a single energy function, we assign to each image its own energy function. The reason we are able to do this is because we model the dependance between images implicitly via the common appearance model $(\mathcal{A})$, while previous works added an explicit histogram matching term to the common energy function. There are two distinct advantages of our approach. First, as several authors [13, 20,21,23] have pointed out, adding an explicit histogram matching term makes the energy function intractable. On the other hand, each one of our energy functions is submodular and can be solved with a single graph- cut. Second, this common energy function grows at least quadratically with the number of images in the group, making these approaches almost impossible to scale to dozens of images in a group. On the other hand, given the appearance models, our collection of energy functions are completely independent. Thus the size of our problem only grows linearly in the number of images in the group, which is critical for interactive applications. In fact, each one of our energy functions may be optimized in parallel, making our approach amenable to distributed systems and multi-core architectures. Videos embedded on our project website [3] show our (single-core) implementation co-segmenting $\sim 20$ image in a matter of seconds.

To be fair, we should note that what allows us to set-up an efficiently solvable energy function is our incorporation of a user in the co-segmentation process, giving us partially labelled data (scribbles). While this user involvement is necessary because we work with globally related images, this involvement also means that the co-segmentation algorithm must be able to query/guide user scribbles, because users cannot be expected to examine all cutouts at each iteration. This is described next.

\section{4. iCoseg: Guiding User Scribbles}

In this section, we develop an intelligent recommendation algorithm to automatically seek user-scribbles and reduce the user effort. Given a set of initial scribbles from the user, we compute a recommendation map for each image in the group. The image (and region) with the highest recommendation score is presented to the user to receive more scribbles. Instead of committing to a single confusion measure as our recommendation score, which might be noisy, we use a number of "cues". These cues are then combined to form a final recommendation map, as seen in Figure 3. The three categories of cues we use, and our approach to learning the weights of the combination are described next.

\subsection{Uncertainty-based Cues}

Node Uncertianty (NU). Our first cue is the one most commonly used in uncertainty sampling, i.e., entropy of the node beliefs. Recall that each time scribbles are received, we fit $A_{1}=\left\{\mathrm{GMM}_{f}, \mathrm{GMM}_{b}\right\}$ to the labelled superpixel features. Using this learnt $A_{1}$, for each superpixel we normalize the foreground and background likelihoods to get a 2-class distribution and then compute the entropy of this distribution. The intuition behind this cue is that the more uniform the class distribution for a site, the more we would like to observe its label.

Edge Uncertainty (EU). The Query by Committee [26] algorithm is a fundamental work that forms the basis for many selective sampling works. The simple but elegant idea is to feed unlabelled data-points to a committee/set of classifiers 
and request label for the data-point with maximal disagreement among classifier outcomes. We use this intuition to define our next cue. For each superpixel, we use our learnt distances (recall: these are used to define the edge smoothness terms in our energy function) to find $K(=10)$ nearest neighbours from the labelled superpixels. We treat the proportion of each class in the returned list as the probability of assigning that class to this site, and use the entropy of this distribution as our cue. The intuition behind this cue is that the more uniform this distribution, the more disagreement there is among the the returned neighbour labels, and the more we would like to observe the label of this site.

Graph-cut Uncertainty (GC). This cue tries to capture the confidence in the energy minimizing state returned by graph-cuts. For each site, we compute the increase in energy by flipping the optimal assignment at that site. The intuition behind this cue is that the smaller the energy difference by flipping the optimal assignment at a site, the more uncertain the system is of its label. We note that minmarginals proposed by Kohli et al. [16] could also be used.

\subsection{Scribble-based Cues}

Distance Transform over Scribbles (DT). For this cue, we compute the distance of every pixel to the nearest scribble location. The intuition behind this (weak) cue is that we would like to explore regions in the image away from the current scribble because they hold potentially different features than sites closer to the current scribbles.

Intervening Contours over Scribbles (IC). This cue uses the idea of intervening contours [18]. The value of this cue at each pixel is the maximum edge magnitude in the straight line to the closest scribble. This results in low confusions as we move away from a scribble until a strong edge is observed, and then higher confusions on the other side of the edge. The motivation behind this cue is that edges in images typically denote contrast change, and by observing scribble labels on both sides of an edge, we can learn whether or not to respect such edges for future segmentations.

\subsection{Image-level Cues}

The cues described so far, are local cues, that describe which region in an image should be scribbled on next. In addition to these, we also use some image-level cues (i.e., uniform over an image), that help predict which image to scribble next, not where.

Segment size (SS). We observe that when very few scribbles are marked, energy minimization methods typically over-smooth and results in "whitewash" segmentations (entire image labelled as foreground or background). This cue incorporates a prior for balanced segmentations by assigning higher confusion scores to images with more skewed segmentations. We normalize the size of foreground and background regions to get class distributions for this image,

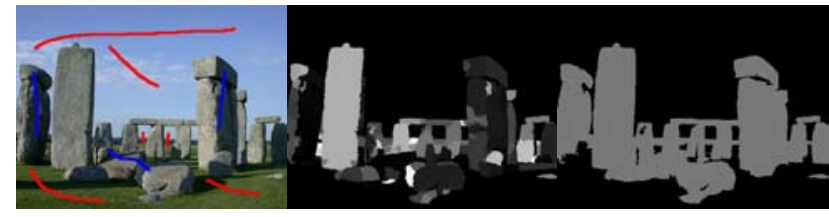

(a) Image+Scribbles

(b) Node Uncertainty

(c) Edge Uncertainty

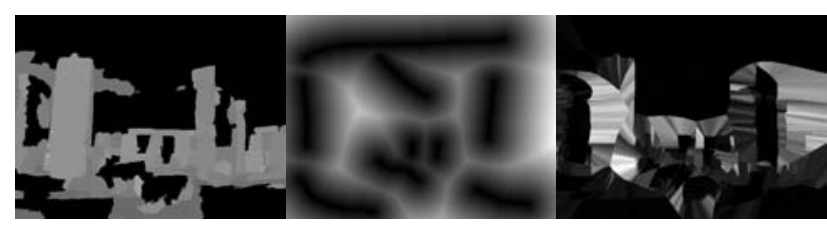

(d) GC Uncertainty

(e) Distance Transform (f) Intervening Contour
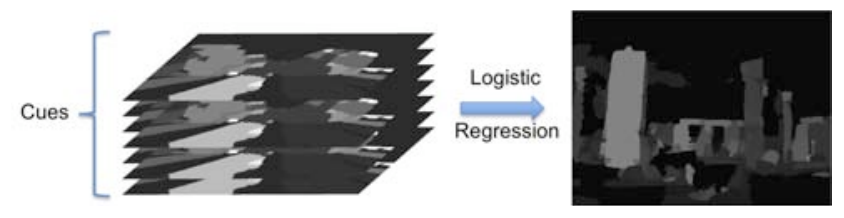

(g) Combining cues

Figure 3: Cues: (a) shows the image with provided scribbles; (b)(f) show various cues; and (g) shows how these cues are combined to produce a final recommendation map.

and use the inverse of the entropy of this distribution as our cue.

Codeword Distribution over Images (CD). This imagelevel cue captures how diverse an image is, with the motivation being that scribbling on images containing more diversity among features would lead to better foreground/background models. To compute this cue, we cluster the features computed from all superpixels in the group to form a codebook, and the confusion score for each image is the entropy of the distribution over the codewords observed in the image. The intuition is that the more uniform the codeword distribution for an image the more diverse the appearances of different regions in the image.

\subsection{Combined Recommendation Map}

We now describe how we combine these various cues to produce a combined confusion map. Intuitively, the optimal combination scheme would be one that generates a recommendation map that assigns high values to regions that a user would scribble on, if they were to exhaustively examine all segmentations. Users typically scribble on regions that are incorrectly segmented. We cast the problem of learning the optimal set of weights for our cues, as that of learning a linear classifier (logistic regression) that maps every superpixel (represented by a 7-dimensional feature vector corresponding to each of the 7 cues described above) to the (binary) segmentation error-map. Our cue combination 
scheme is illustrated in Figure 3.

\section{The CMU-Cornell iCoseg Dataset}

To evaluate our proposed approach and to establish a benchmark for future work, we introduce the largest cosegmentation dataset yet, the CMU-Cornell iCoseg Dataset. While previous works have experimented with a few pairs of images, our dataset contains 38 challenging groups with 643 total images ( $\sim 17$ images per group), with associated pixel-level ground truth. We built this dataset from the Flickr® online photo collection, and hand-labelled pixellevel segmentations in all images. We used the "Group" feature in Flickr, where users form groups around popular themes, to search for images from this theme. Our dataset consists of animals in the wild (elephants, pandas, etc.), popular landmarks (Taj Mahal, Stonehenge, etc.), sports teams (Baseball, Football, etc.) and other groups that contain a common theme or common foreground object. For some (though not all) of the groups, we restricted the images to come from the same photographer s photo-stream, making this a more realistic scenario. Examples of these groups are shown in various figures in this paper and more examples may be found online [3]. We note that this dataset is significantly larger than those used in previous works $[13,23]$. We have made this dataset (and annotations) publicly available [3] to facilitate further work, and allow for easy comparisons.

\section{Experiments}

\subsection{Machine Experiments}

To conduct a thorough set of experiments and evaluate various design choices, it is important to be able to perform multiple iterations without explicitly polling a human for scribbles. Thus, we develop a mechanism to generate automatic scribbles, that mimic human scribbles (we also present results of a user-study in Section 6.2). We model these synthetic scribbles as (smooth) random walks that do not cross foreground-background boundaries. Our scribble generation technique consists of sampling a starting point in the image uniformly at random. A direction angle is then randomly sampled such that it is highly correlated with the previous direction sample (for smoothness) for the scribble, ${ }^{3}$ and a fixed-size (=30 pixels) step is taken along this direction to extend the scribble (as long as it does not cross object boundaries, as indicated by the groundtruth segmentation of the image). To mimic user-scribbles given a recommendation map, the initial as well as subsequent points on the scribble are picked by considering the recommendation map to be a distribution. Using synthetic scribbles

\footnotetext{
${ }^{3}$ For the first two sampled points, there is no previous direction and this direction is sampled uniformly at random.
}

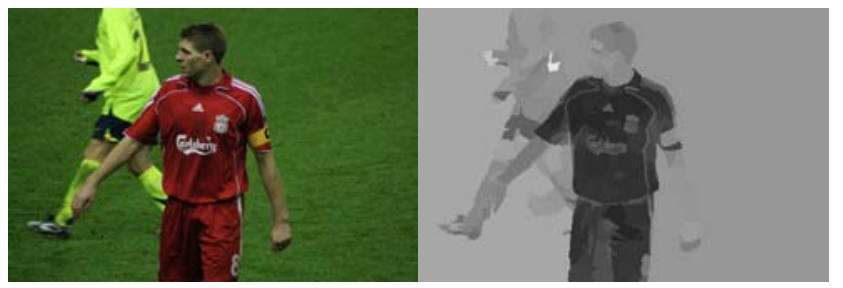

(a) Image

(b) Confusion Map

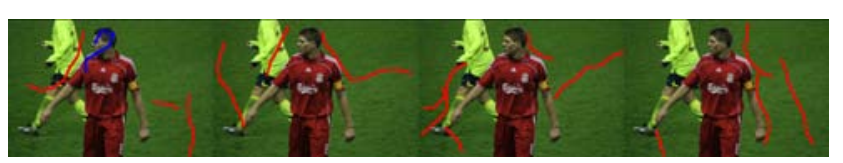

(c) Sampled Scribbles

Figure 4: Example simulated scribbles: Note that these scribbles never cross foreground boundaries (red player).

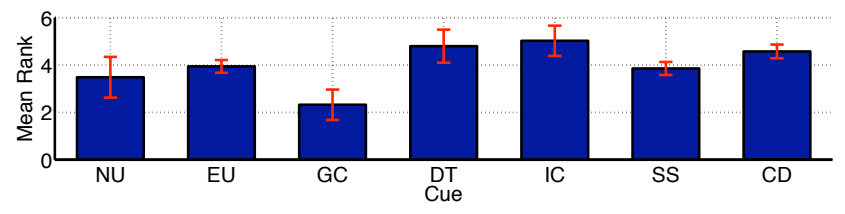

Figure 5: Mean ranks achieved by individual cues (see Sec 6.1).

allows us to control the length of scribbles and observe the behavior of the algorithm with increasing information. Example synthetic scribbles are shown in Figure 4.

We first analyze the informativeness of each of our 7 cues. We start by generating a foreground and background scribble on a random image in a group. We then compute each of our cues, and treat each individual cue as a recommendation map. We generate the next synthetic scribble as guided by this recommendation map. We repeat this till we have scribbled about 1000 pixels across the group, and compute the average segmentation accuracy across the images of a group. ${ }^{4}$ We rank the 7 cues by this accuracy. Figure 5 shows the mean ranks (across groups, average of 10 random runs) achieved by these cues. Out of our cues, the graph-cut cue (GC) performs the best, while both distance transform (DT) and intervening contour (IC) are the weakest.

We now evaluate iCoseg, our recommendation system, as a whole. The experimental set up is the same as that described above, except now we use the combined recommendation map to guide subsequent scribbles (and not individual cues). The cue combination weights are learnt from all groups except one that we test on (leave-one-out cross validation). We compare to two baselines. One is that of using a uniform recommendation map on all images in the

\footnotetext{
${ }^{4}$ In order to keep statistics comparable across groups, we select a random subset of 5 images from all groups in our dataset. One of our groups consisted of 4 images only, so all our results are reported on 37 groups.
} 


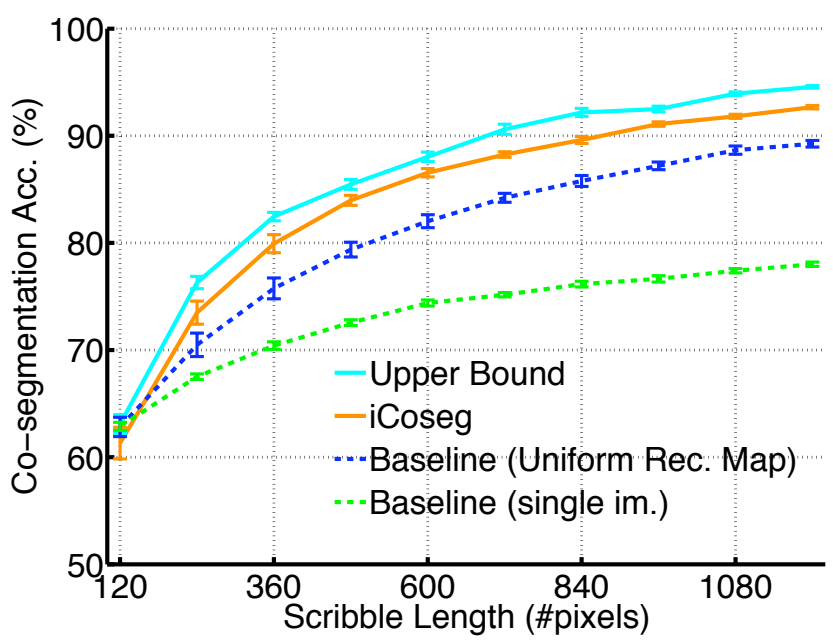

Figure 6: Machine Experiments: iCoseg significantly outperforms baselines and is close to a natural upper-bound (Section 6.1).

group, which essentially means randomly scribbling on the images (respecting object boundaries of course). And the other (even weaker) baseline is that of selecting only one image (randomly) in a group to scribble on (with a uniform recommendation map on this image).

Figure 6 shows the performance of our combined recommendation map (iCoseg) with increasing scribble length, as compared to the baselines. We see that our proposed recommendation scheme does in fact provide meaningful guidance for regions to be scribbled on next (as compared to the two baselines). A meaningful upper-bound would be the segmentation accuracy that could be achieved if an oracle told us where the segmentations were incorrect, and subsequent scribbles were provided only in these erroneous regions. As seen in Figure 6, iCoseg performs very close to this upper bound, which means that users following our recommendations can achieve cutout performances comparable to those achieved by analyzing mistakes in all cutouts with significantly less effort without ever having to examine all cutouts explicitly.

\subsection{User Study}

In order to further test iCoseg, we developed a java-based user-interface for interactive co-segmentation. ${ }^{5}$ We conducted a user study to verify our hypothesis that our proposed approach can help real users produce good quality cutouts from a group of images, without needing to exhaustively examine mistakes in all images at each iteration. Our study involved 15 participants performing 3 experiments (each involving 5 groups of 5 related images). Figure 8 shows screen-shots from the three experiments. The sub-

\footnotetext{
${ }^{5}$ We believe this interface may be useful to other researchers working on interactive applications and we have made it publicly available [3].
}

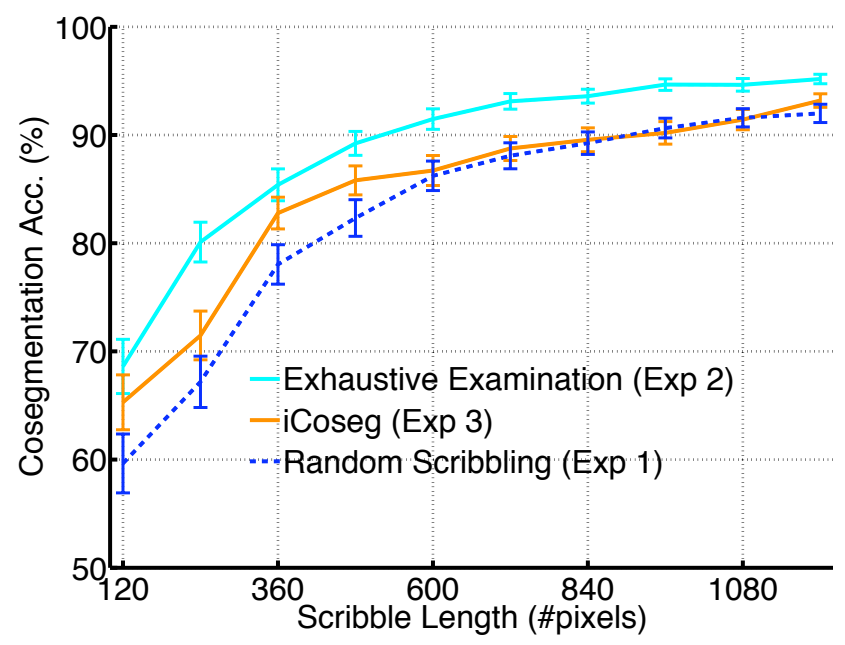

Figure 7: User Study: Average performance of subjects in the three conducted experiments (see Section 6.2). iCoseg (Exp. 3) requires significantly less effort for users, e.g. allowing them to reach $80 \%$ co-seg accuracy with three-fourth the effort of Exp. 1.

jects were informed that the first experiment was to acclimatize them to the system. They could scribble anywhere on any image, as long as they used blue scribbles on foreground and red scribbles on background. The system computed cutouts based on their scribbles, but the subjects were never shown these cutouts. We consider this experiment to be a replica of the random-scribble setup, thus forming a lower bound for the active learning setup. In the second experiment, the subjects were shown the cutouts produced produced on all images in the group from their scribbles. Their goal was to achieve 95\% co-segmentation accuracy in as few interactions as possible, and they could scribble on any image. We observed that a typical strategy used by subjects was to find the worst cutout at every iteration, and then add scribbles to correct it. In the third experiment, they had the same goal, but this time, while were shown all cutouts, they were constrained to scribble within a window recommended by our algorithm, iCoseg. This window position was chosen by finding the location with the highest average recommendation value (in the combined recommendation map) in a neighbourhood of $201 \times 201$ pixels. The use of a window was merely to make the user-interface intuitive, and other choices could be explored.

Figure 7 shows the average segmentation accuracies achieved by the subjects in the three experiments. We can see that, as with the machine experiments, iCoseg helps the users perform better than random scribbling, in that the same segmentation accuracy (80\%) can be achieved with about three-fouth the effort (in terms of length of scribbles). In addition, the average time taken by the users for one iteration of scribbling reduced from 20.2 seconds (exhaustively examining all cutouts) to 14.2 seconds (iCoseg), an aver- 


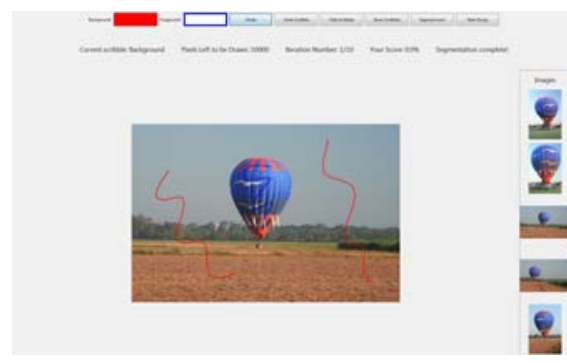

(a) Experiment 1

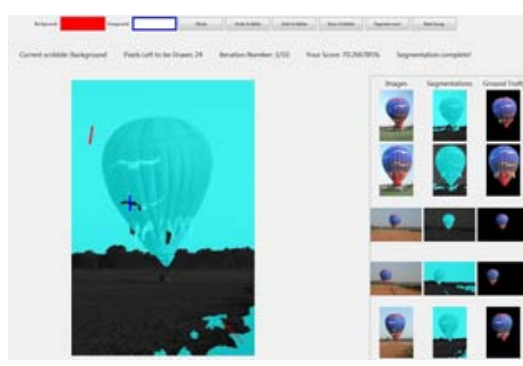

(b) Experiment 2

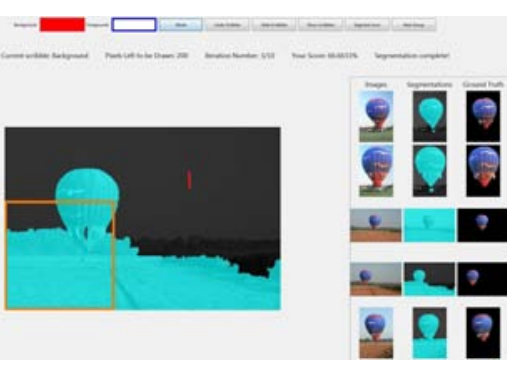

(c) Experiment 3

Figure 8: User Study Screenshots: (a) Exp. 1: subjects were not shown cutouts and were free to scribble on any image/region while respecting the foreground/background boundaries; (b) Exp. 2: subjects exhaustively examine all segmentations and scribble on mistakes (cyan indicates foreground); (c) Exp. 3: users were instructed to scribble in the region recommended by iCoseg. Best viewed in colour.

age saving of 60 seconds per group. Thus, our approach enables users to achieve cutout accuracies comparable to those achieved by analyzing mistakes in all cutouts, in significantly less time.

\section{Conclusions}

We present an algorithm for interactive co-segmentation of a group of realistic related images. We propose iCoseg, an approach that co-segments all images in the group using an energy minimization framework, and an automatic recommendation system that intelligently recommends a region among all images in the group where the user should scribble next. We introduce and make publicly available the largest co-segmentation dataset, the CMU-Cornell iCoseg dataset containing 38 groups (643 images), along with pixel groundtruth hand annotations [3]. In addition to machine experiments with synthetic scribbles, we perform a userstudy on our developed interactive co-segmentation interface (also available online), both of which demonstrate that using iCoseg, users can achieve good quality segmentations with significantly lower time and effort than exhaustively examining all cutouts.

Acknowledgements: The authors would like to thank YuWei Chao for data collection and annotation, and Kevin Tang for developing the java-based GUI (iScrrible) [3] used in our user-studies.

\section{References}

[1] S. Bagon. Matlab wrapper for graph cut, December 2006.

[2] X. Bai and G. Sapiro. A geodesic framework for fast interactive image and video segmentation and matting. In ICCV, 2007.

[3] D. Batra, A. Kowdle, D. Parikh, K. Tang, and T. Chen. http: //amp.ece.cornell.edu/projects/touch-coseg/, 2009. Interactive Cosegmentation by Touch.

[4] D. Batra, R. Sukthankar, and T. Chen. Semi-supervised clustering via learnt codeword distances. In $B M V C, 2008$.

[5] C. A. Bouman. Cluster: An unsupervised algorithm for modeling Gaussian mixtures. Available from http://www.ece.purdue.edu/'bouman, April 1997.

[6] Y. Boykov and M.-P. Jolly. Interactive graph cuts for optimal boundary and region segmentation of objects in n-d images. ICCV, 2001.
[7] Y. Boykov and V. Kolmogorov. An experimental comparison of mincut/max-flow algorithms for energy minimization in vision. PAMI, 26(9):1124-1137, 2004.

[8] Y. Boykov, O. Veksler, and R. Zabih. Efficient approximate energy minimization via graph cuts. PAMI, 20(12):1222-1239, 2001.

[9] B. Collins, J. Deng, K. Li, and L. Fei-Fei. Towards scalable dataset construction: An active learning approach. In ECCV, 2008.

[10] D. Comaniciu and P. Meer. Mean shift: a robust approach toward feature space analysis. PAMI, 24(5):603-619, 2002.

[11] A. Criminisi, T. Sharp, and A. Blake. Geos: Geodesic image segmentation. In ECCV, 2008.

[12] J. Cui, Q. Yang, F. Wen, Q. Wu, C. Zhang, L. V. Gool, and X. Tang. Transductive object cutout. In $C V P R, 2008$.

[13] D. S. Hochbaum and V. Singh. An efficient algorithm for cosegmentation. In ICCV, 2009.

[14] D. Hoiem, A. A. Efros, and M. Hebert. Geometric context from a single image. In ICCV, 2005.

[15] A. Kapoor, K. Grauman, R. Urtasun, and T. Darrell. Active learning with gaussian processes for object categorization. In ICCV, 2007.

[16] P. Kohli and P. H. S. Torr. Measuring uncertainty in graph cut solutions. CVIU, 112(1):30-38, 2008.

[17] V. Kolmogorov and R. Zabih. What energy functions can be minimized via graph cuts? PAMI, 26(2):147-159, 2004.

[18] T. Leung and J. Malik. Contour continuity in region based image segmentation. In ECCV, 1998.

[19] Y. Li, J. Sun, C.-K. Tang, and H.-Y. Shum. Lazy snapping. SIGGRAPH, 2004.

[20] Y. Mu and B. Zhou. Co-segmentation of image pairs with quadratic global constraint in mrfs. In $A C C V, 2007$.

[21] L. Mukherjee, V. Singh, and C. R. Dyer. Half-integrality based algorithms for cosegmentation of images. In CVPR, 2009.

[22] C. Rother, V. Kolmogorov, and A. Blake. "Grabcut": interactive foreground extraction using iterated graph cuts. SIGGRAPH, 2004.

[23] C. Rother, T. Minka, A. Blake, and V. Kolmogorov. Cosegmentation of image pairs by histogram matching - incorporating a global constraint into mrfs. In $C V P R, 2006$.

[24] Y. Schnitman, Y. Caspi, D. Cohen Or, and D. Lischinski. Inducing semantic segmentation from an example. In ACCV, 2006.

[25] B. Settles. Active learning literature survey. Computer Sciences Technical Report 1648, University of Wisconsin-Madison, 2009.

[26] H. S. Seung, M. Opper, and H. Sompolinsky. Query by committee. In COLT, 1992.

[27] S. Vijayanarasimhan and K. Grauman. What s it going to cost you?: Predicting effort vs. informativeness for multi-label image annotations. In $C V P R, 2009$.

[28] R. Yan, J. Yang, and A. Hauptmann. Automatically labeling video data using multi-class active learning. In ICCV, 2003. 